\title{
Acute Rupture of Sinus of Valsalva into Right Atrium: An Echocardiographic Halftone
}

\author{
Neeti Makhija ${ }^{1}$ Devishree Das ${ }^{1} \quad$ Sumit Agarwal ${ }^{1}$ \\ ${ }^{1}$ Department of Cardiac Anaesthesia, Cardiothoracic and \\ Neurosciences Centre (CNC), All India Institute of Medical Sciences, \\ New Delhi, India \\ J Card Crit Care 2021;5:252-256.
}

\begin{abstract}
Address for correspondence Neeti Makhija, MD, Department of Cardiac Anaesthesia, Cardiothoracic and Neurosciences Centre, All India Institute of Medical Sciences, Room No. 9, 7th Floor, Ansari Nagar, New Delhi 110029, India (e-mail: neetimakhija@hotmail.com).
\end{abstract}

\author{
Abstract \\ Keywords \\ - aortic rupture \\ - echocardiography \\ - ruptured sinus of \\ Valsalva \\ - sinus of Valsalva \\ - transesophageal \\ echocardiography
}

A ruptured sinus of Valsalva is a rare and emergent cardiac abnormality, which can be acute and rapidly expanding to cause hemodynamic deterioration. Rupturing into right-sided heart chambers causes severe left-to-right shunt and sudden volume overload. Echocardiography especially transesophageal echocardiography (TEE) is the gold standard facility for early perioperative diagnosis and also to guide surgical technique. Medical stabilization followed by prompt surgical intervention is the treatment of choice.

We hereby report a case of sinus of Valsalva rupturing into right atrium leading to severe left-to-right shunt and the role of TEE in its perioperative management.

\section{Introduction}

Sinus of Valsalva aneurysm (SOVA) is an abnormal aneurysmal dilatation of the aortic sinus of Valsalva. It is located between the aortic annulus and sinotubular junction. The dilatation occurs due to weakness of elastic tissue present at the junction of the aortic media and the fibrous annulus. ${ }^{1}$ Aneurysms of the sinus of Valsalva are typically more common in men (4:1) and there is a higher incidence in Asian populations. ${ }^{2}$ Ruptured sinus of Valsalva (RSOV) is a potentially fatal consequence of SOVA and the patient should be stabilized medically but mandate urgent surgical repair. Transesophageal echocardiography (TEE) is an useful tool in the perioperative setting., ${ }^{3,4}$ It not only diagnose the SOVA, but also determines its extension, the chamber where it ruptures, and associated defects, thus guiding the surgical technique.

We report a case of sinus of Valsalva rupturing into right atrium where patient was stabilized medically followed by surgical repair and the utility of perioperative echocardiography.

\section{Case Report}

A 48-year-old male presented with sudden onset dragging type chest pain in the lower retrosternal and epigastric region after moderate physical exertion. The patient developed progressive dyspnea and orthopnea. On examination patient had raised jugular venous pressure, pedal edema, and tender hepatomegaly. On auscultation, a continuous machinery cardiac murmur of grade $4 / 6$ was heard at left parasternal border and crepitations in bilateral basal lung fields. Chest Xray showed features of pulmonary edema. Transthoracic echocardiography demonstrated RSOV of noncoronary cusp into right atrium causing left-to-right shunt, mild aortic regurgitation with normal ejection fraction. Computed tomographic aortogram revealed aneurysmal dilatation of noncoronary sinus with $17 \mathrm{~mm}$ opening into right atrium.
DOI https://doi.org/ 10.1055/s-0041-1741493. ISSN 2457-0206. (c) 2022. Official Publication of The Simulation Society (TSS), accredited by International Society of Cardiovascular Ultrasound (ISCU). All rights reserved.

This is an open access article published by Thieme under the terms of the Creative Commons Attribution-NonDerivative-NonCommercial-License, permitting copying and reproduction so long as the original work is given appropriate credit. Contents may not be used for commercial purposes, or adapted, remixed, transformed or built upon. (https://creativecommons.org/ licenses/by-nc-nd/4.0/)

Thieme Medical and Scientific Publishers Pvt. Ltd., A-12, 2nd Floor, Sector 2, Noida-201301 UP, India 
Diuretics and angiotensin-converting enzyme inhibitors were started to treat the acute episode and patient was planned for surgical intervention. In the operating room, standard American Society of Anaesthesiologists (ASA) monitors were attached and large bore peripheral venous line and arterial access were secured under local anesthesia. The anesthesia was induced with titrated dose of midazolam, fentanyl, etomidate, and muscle relaxant cisatracurium. After endotracheal intubation, temperature probe and TEE probe (PHILIPS X7-2t Adult) were placed and central venous line secured in the right internal jugular vein.

The TEE was performed using Philips iE33 (Bothell, Washington, United States) machine and X7-2t Adult TEE probe $(2-6 \mathrm{MHz})$. On mid-esophageal 4-chamber (right chambers focused) view, aneurysmal sinus was found to be draining into right atrium and flow turbulence was noted on color comparison (-Fig. 1). The mid-esophageal right ventricle inflow-outflow view showed RSOV draining into right atrium with characteristic windshock appearance (-Fig. 2). RSOV with windshock could also be demonstrated in the deep transgastric view (-Fig. 3). Trivial aortic regurgitation with normal biventricular function was found. There were no other associated anomalies. The aneurysmal sac was resected and the noncoronary cusp was augmented with patch which was confirmed in the mid-esophageal aortic valve short-axis view (-Fig. 4). Aortic valve morphology restored with normal leaflet motion, good coaptation, and without aortic regurgitation. The cardiopulmonary bypass (CPB) and aortic cross-clamp time were 38 and 19 minutes, respectively. After repair, the patient was weaned from CPB with infusion nitroglycerine 0.5 $\mathrm{mcg} / \mathrm{kg} / \mathrm{min}$. Adequate hemostasis was achieved and the patient was shifted to intensive care unit for postoperative management. The CPB and postoperative management was as per institutional protocol. After 3 hours of mechanical ventilation patient was extubated. The postoperative course was uneventful.

\section{Discussion}

The rare cardiac anomaly, SOVA, can be of congenital or acquired origin. The congenital causes for SOVA were associated mostly with Marfan syndrome, Ehlers-Danlos syndrome, and other connective tissue disorders. ${ }^{1}$ Physical exertion, trauma, infective endocarditis, atherosclerosis, and iatrogenic injury during cardiac surgery constitute acquired causes. SOVA most commonly arise from right coronary sinus (incidence 70\%), followed by noncoronary sinus

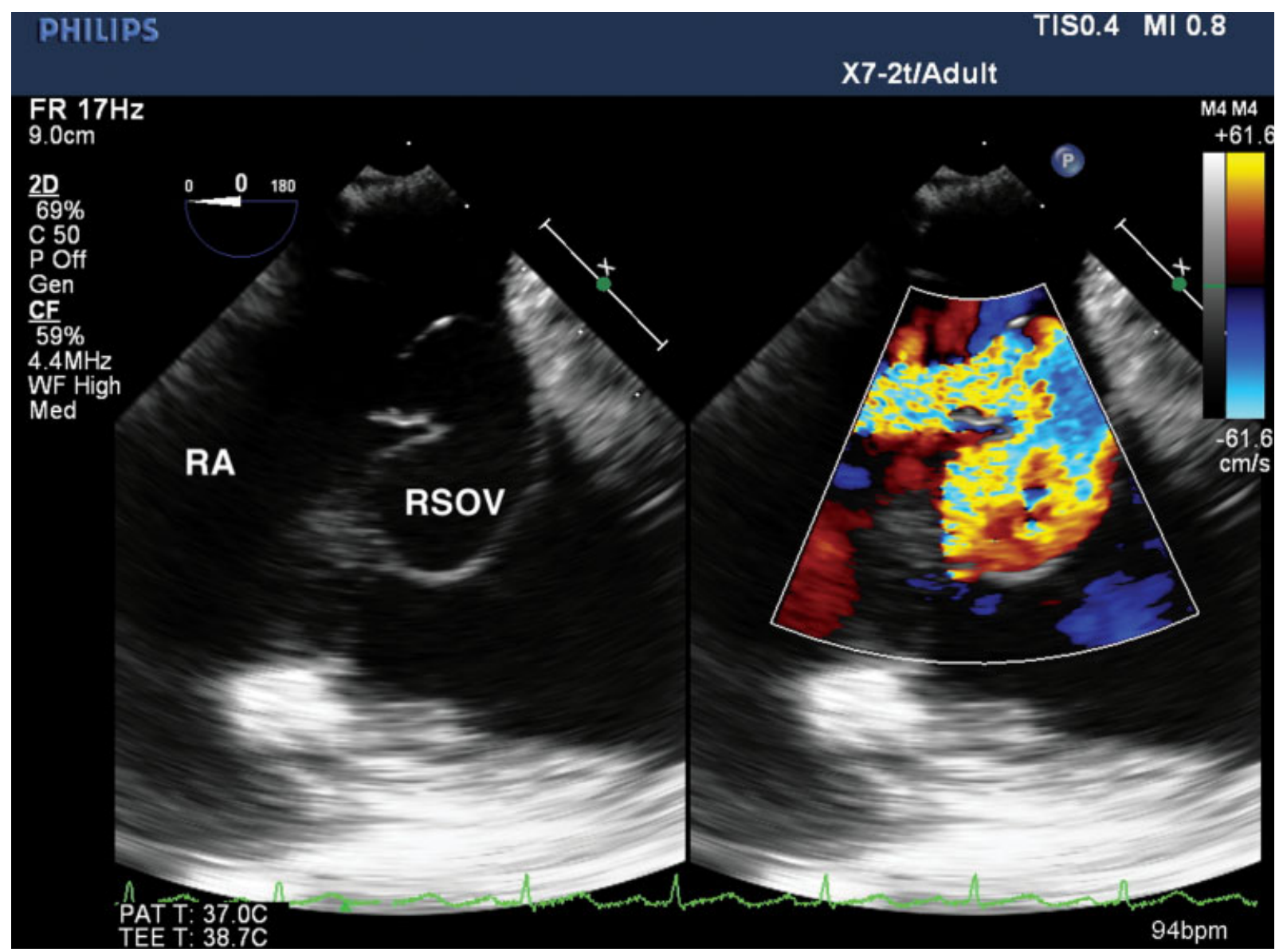

Fig. 1 Two-dimensional transesophageal echocardiography (2D-TEE) mid-esophageal 4-chamber color compare view (right chamber focused) showing aneurysmal sinus (ruptured sinus of Valsalva [RSOV]) draining into right atrium (RA) with flow turbulence. 


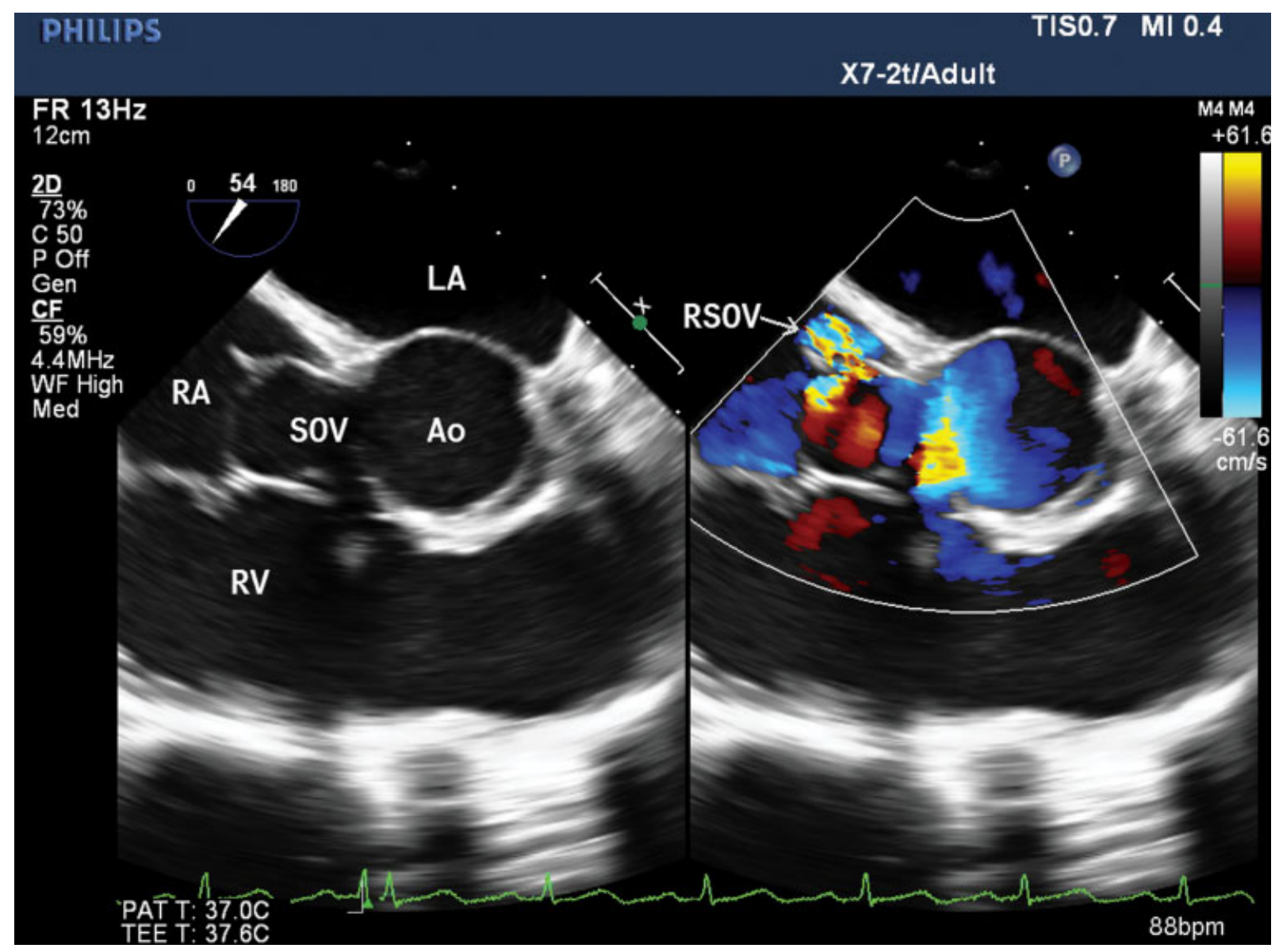

Fig. 2 Two-dimensional transesophageal echocardiography (2D-TEE) mid-esophageal right ventricle (RV) inflow-outflow view with color comparison revealing sinus of Valsalva (SOV) draining into right atrium (RA) with characteristic windshock appearance. LA, left atrium; RV, right ventricle; $\mathrm{AO}$, aorta.

(25\%). Therefore, if it ruptures a left-to-right shunt develops into either right ventricle or right atrium. ${ }^{5}$ Also, cases of right ventricular outflow obstruction, coronary artery compression, thrombus formation, and conduction disturbances have been reported. ${ }^{6}$

The most common complication of SOVA is rupture. Rarely, it may rupture into left atrium, left ventricle, pulmonary artery, or pericardial cavity. Traditionally, echocardiography, especially transthoracic modality has been the first-line technique. Other imaging techniques include multislice computed tomography and magnetic resonance imaging. ${ }^{7}$ However, TEE has become the gold standard imaging technique where its anatomy, extension, valvular involvement, and any other cardiac anomalies can be precisely detected. Color Doppler interrogation will show continuous flow in both systole as well as in diastole. TEE can demonstrate acute aortic regurgitation, morphology of aortic valve, involved chamber dimensions, and cardiac function. TEE also differentiates it from other similar looking abnormalities like aortic root abscess which may be due to infective endocarditis and may not communicate with cardiac chambers. ${ }^{8}$ These were the corner stone for perioperative management. If SOVA is associated with coronary artery anomalies TEE confirm the pattern of anomaly, guide cardioplegia flow, and detect postrepair coronary injury as regional wall motion abnormalities. TEE plays a crucial role in guiding repair of valvular apparatus, and other cardiac and extracardiac structures associated with SOVA. Also, postsurgical repair, the adequacy of repair, coronary patency, and valve competency can be determined. Newer modalities like three-dimensional (3D) TEE not only provide better delineation of RSOV but also accurately demonstrate its extent, exact size, and site of rupture. Therefore, 3D TEE represents as an adjunctive tool in surgical as well as transcatheter closure of RSOV. ${ }^{9}$

The median survival of RSOV is 3.9 years, if left untreated which mandates early surgical intervention. Congestive heart failure is the main cause of mortality. An open-heart surgery is the main treatment option. Moreover, percutaneous transcatheter closure of RSOV has been reported. ${ }^{10}$ Surgical treatment technique include primary closure, patch repair, or aortic root replacement with or without valve replacement. Perioperative mortality is between 1.9 and 3.9\%. ${ }^{5}$ After repair, patient's life expectancy approximates that of the healthy population. 


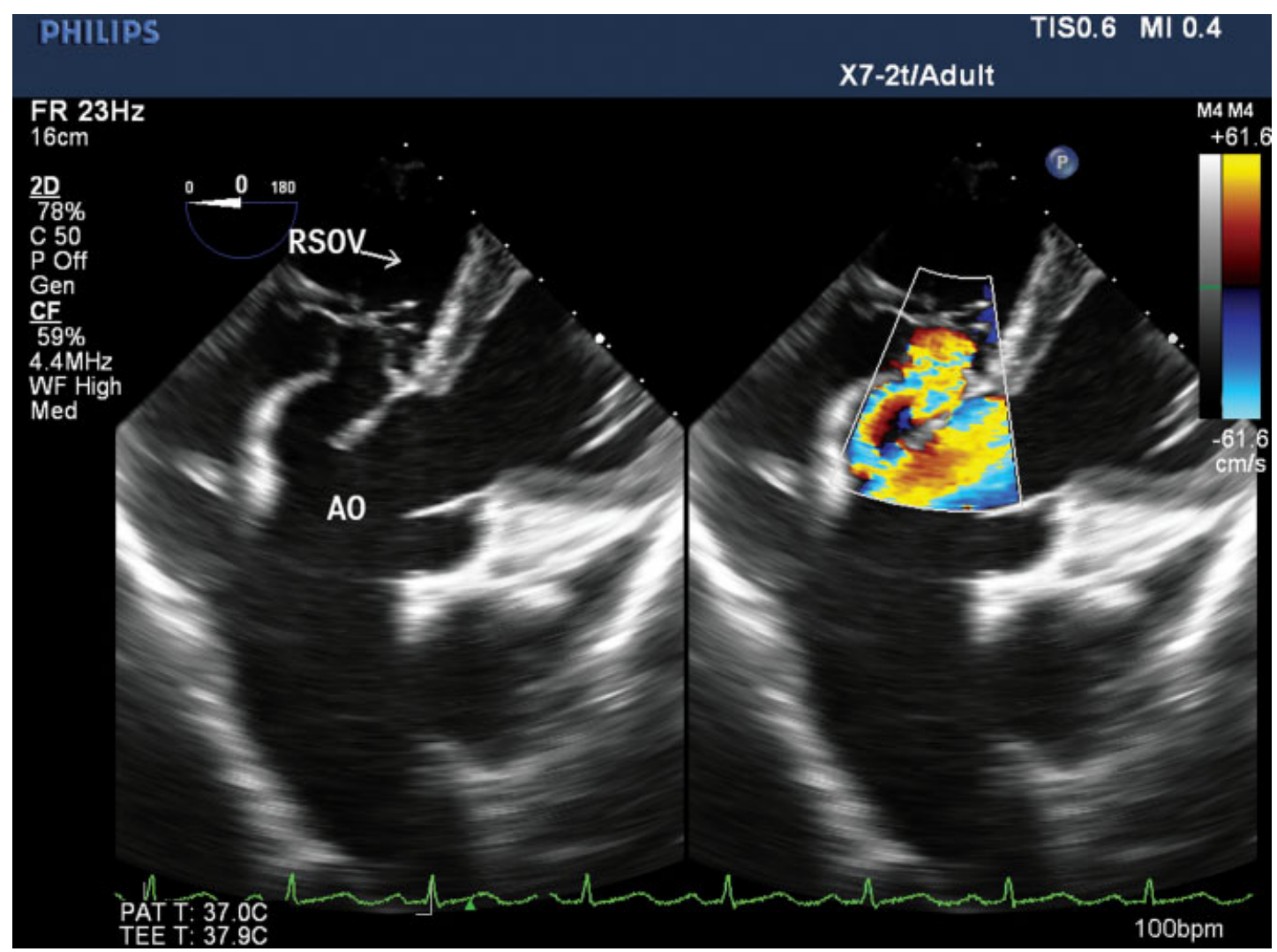

Fig. 3 Two-dimensional transesophageal echocardiography (2D-TEE) deep transgastric view with color comparison confirming rupture sinus of Valsalva (RSOV) and normal aorta (AO).

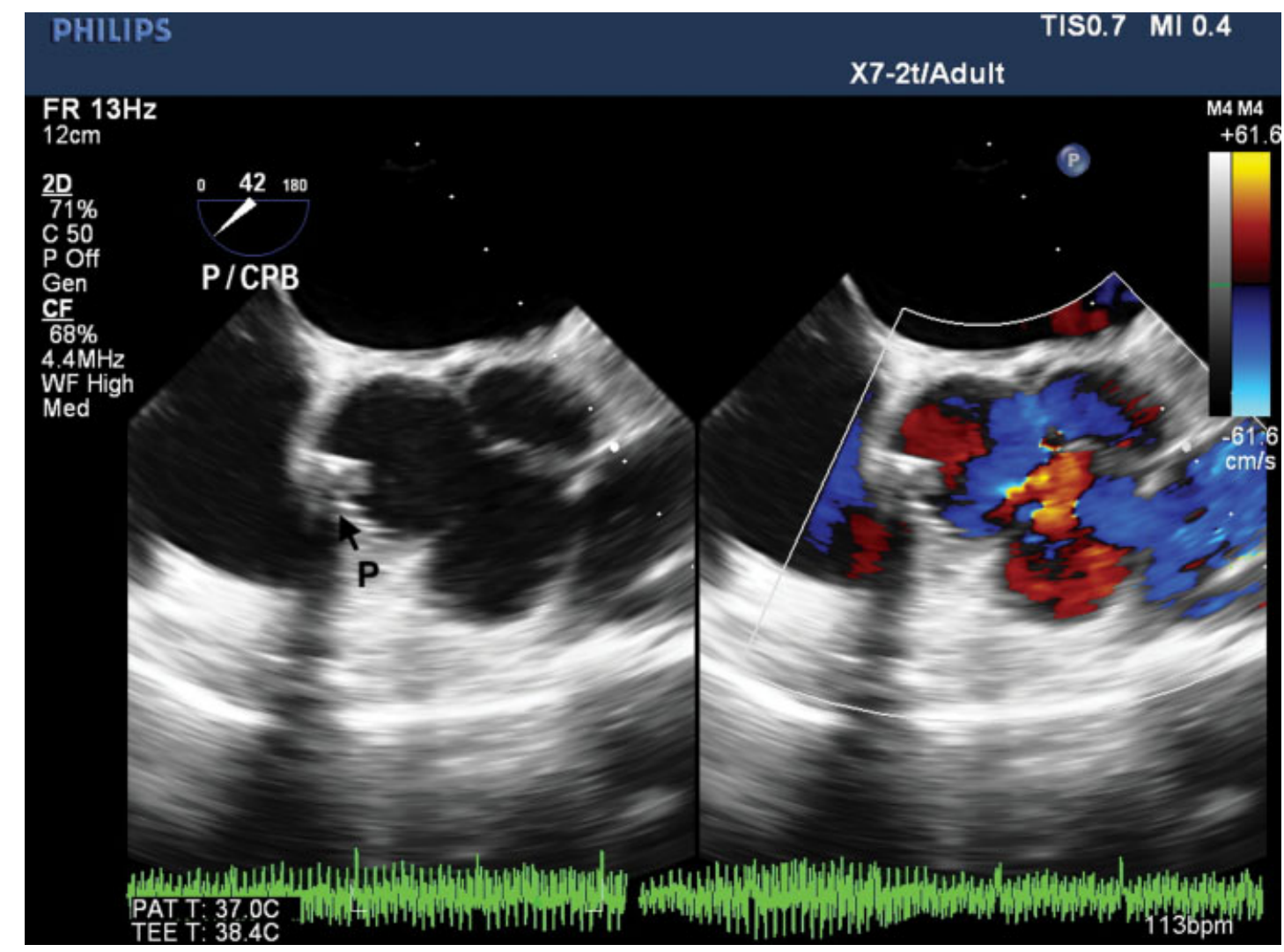

Fig. 4 Two-dimensional transesophageal echocardiography (2D-TEE) mid-esophageal aortic valve short-axis view with color comparison demonstrating normal aortic valve and the patch $(P)$. 


\section{Conclusion}

SOVAs can be asymptomatic until it ruptures. Rupture is the dreaded complication of SOVA and it progress rapidly endangering life. Echocardiography is an excellent imaging modality, as it not only facilitates timely diagnosis but also determines other associated abnormalities. TEE is the perioperative guiding tool for adequate surgical intervention.

\section{Conflict of Interest \\ None declared.}

\section{References}

1 Diwakar A, Patnaik SS, Hiremath CS, Chalam KS, Dash P. Rupture of sinus of valsalva - a 15 years single institutional retrospective review: preoperative heart failure has an impact on post operative outcome? Ann Card Anaesth 2019;22(01):24-29

2 Feldman DN, Roman MJ. Aneurysms of the sinuses of Valsalva. Cardiology 2006;106(02):73-81

3 Serban AM, Bătrâna N, Cocoi M, et al. The role of echocardiography in the diagnosis and management of a giant unruptured sinus of Valsalva aneurysm. Med Ultrason 2019;21(02):194-196
4 Wierda E, Koolbergen DR, de Mol BAJM, Bouma BJ. Rupture of a giant aneurysm of the sinus of Valsalva leading to acute heart failure: a case report demonstrating the excellence of echocardiography. Eur Heart J Case Rep 2018;2(03):yty090

5 Moustafa S, Mookadam F, Cooper L, et al. Sinus of Valsalva aneurysms -47 years of a single center experience and systematic overview of published reports. Am J Cardiol 2007;99(08): 1159-1164

6 Vural KM, Şener E, Taşdemir O, Bayazit K. Approach to sinus of Valsalva aneurysms: a review of 53 cases. Eur J Cardiothorac Surg 2001;20(01):71-76

7 Bricker AO, Avutu B, Mohammed T-LH, et al. Valsalva sinus aneurysms: findings at $\mathrm{CT}$ and MR imaging. Radiographics 2010;30(01):99-110

8 Shah S, Murray H. Potential chronic abscess cavity that masqueraded as sinus of Valsalva aneurysm: is TEE a vital tool? Heart Lung Vessel 2014;6(02):130-134

9 Kumar GA, Parimala PS, Jayaranganath M, Jagadeesh AM. Threedimensional transesophageal echocardiography-guided transcathetar closure of ruptured noncoronary sinus of valsalva aneurysm. Ann Card Anaesth 2017;20(Supplement):S73-S75

10 Kerkar PG, Lanjewar CP, Mishra N, Nyayadhish P, Mammen I. Transcatheter closure of ruptured sinus of Valsalva aneurysm using the Amplatzer duct occluder: immediate results and midterm follow-up. Eur Heart J 2010;31(23):2881-2887 